\begin{tabular}{l} 
SCIENCE \& TECHNOLOGY \\
Journal homepage: http://www.pertanika.upm.edu.my/ \\
\hline PERTANIKA
\end{tabular}

\title{
Analytical and Numerical Investigations of Mechanical Vibration in the Vertical Direction of a Human Body in a Driving Vehicle using Biomechanical Vibration Model
}

\author{
Maher Al-Baghdadi ${ }^{1}$, Muhsin Jaber Jweeg ${ }^{2}$ and Muhannad Al-Waily ${ }^{1 *}$ \\ ${ }^{1}$ Mechanical Engineering Department, Faculty of Engineering, University of Kufa, Iraq \\ ${ }^{2}$ College of Technical Engineering, Al-Farahidi University, Iraq
}

\begin{abstract}
The main reason that affects the discomfort in a driving vehicle is the vibration response. The human body vibration leads to many malfunctions in both comfort and performance in human health. As a result, the human body's simulation in sitting posture in the driving vehicle has a strategic relationship for all Tires and vehicles manufacturers. The digital process simulation of the human body seat vehicle vibration shows two significant advantages. The first advantage is the prevention of the high-cost modifications in the construction stage of the vehicle, while the second one describes the stability test during the undesirable vibrations. This study modelled the human body's dynamic characterisations, natural frequency, and mechanical response when seated in the driving vehicle with vibration transmissibility in the vertical direction have been using the biomechanical vibration model. The vertical vibrations and the transmissibility of the human body dynamic response are presented in detail. Exciting results have been obtained, and they are significant for human health, which relates to sitting posture in the driving vehicle. It can assist in understanding the influences of low-frequency vibration on human health, comfort, and performance, and therefore it could be applied for ride comfort evaluation. An analytical solution

ARTICLE INFO

Article history:

Received: 11 April 2021

Accepted: 19 August 2021

Published: 18 October 2021

DOI: https://doi.org/10.47836/pjst.29.4.30

E-mail addresses:

mahirar.albaghdadi@uokufa.edu.iq (Maher Al-Baghdadi) muhsin.jweeg@uoalfarahidi.edu.iq (Muhsin Jaber Jweeg) muhanedl.alwaeli@uokufa.edu.iq (Muhannad Al-Waily)

* Corresponding author

to derive the general equations of motion for the human system was developed. Then, using the vibration analysis technique and the corresponding equations, the accurate dynamic response of the selected mode is identified. Furthermore, the mathematical modelling for free vibration using the finite element analysis has been performed to
\end{abstract}


determine the appropriate values and set its description. Then, the comparison results of the two techniques have been carried out.

Keywords: Apparent mass, biomechanical model, driving vehicle, human body vibration, mechanical response, model analysis

\section{INTRODUCTION}

A vibration problem in a driving vehicle is the main reason for discomfort for passengers. Human body vibration leads to many problems in human health, comfort feeling, and performance. The problems raised due to vibrations and their relations to the human body were investigated by many researchers. Most of the results obtained from these studies were the level of injury, and the discomfort can be estimated with this relationship (ISO 2631-1, 1997). The experiments on the human body's biodynamic responses in a driving vehicle to vertical vibration excitation have been measured and presented in International Standard 5982 (ISO 5982, 2001). The work also covered the investigation of the dynamic body response has different effects for different people, which is related to the inter-subject variability and within the person related to the intra-subject variability. Experimental work was presented and included investigating the factors that influence the human body mass, such as the ratio between the vertical force and the measured acceleration at the supporting seat. The vibration spectra, sitting posture, age, and vibration magnitude (Zheng et al., 2011), many forms of biodynamic models have been developed to represent the driving point apparent mass and the transmissibility of the human body.

The biodynamic models can be classified as lumped and multibody and finite element types. The lumped simulation is employed to predict the modulus and the inline vertical apparent mass phase. This modelling gives a reasonable agreement of the experimental measurement of the frequencies up to $20 \mathrm{~Hz}$ in the case of one degree of freedom systems. It also gives an excellent correlation in modelling two degrees of freedom systems (Mohanty \& Mahapatra, 2014). The movement of the human body segments during vertical excitation can be modelled using the combination of lumped-mass and rigid body dynamic response (Guo et al., 2016). The modelling does not take into considerations all the degrees of freedom of the body segments. It includes the pitching of the upper torso and the translational degrees of freedom of the lower body. For modelling the human body segments, they are represented by rigid bodies interconnected by joints that can be freely rotated (Nimmen et al., 2017).

The improvement of the spine's biomechanical vibration models and the torso opens the door to detect the harmful effects on the spine. The spinal load's effects have been analysed numerically using a multibody model of the human spine to calculate the compressive 
forces at the trunk discs (Bayoglu et al., 2019). In addition, finite element biomechanical models have been used to simulate the human torso to evaluate the effectiveness of brace design in the context of adolescent idiopathic scoliosis on large cohorts of patients (Vergari et al., 2020).

The sensitivity and importance of the biomechanical vibration models have been recently focused on because of their importance in designing the seats of different modes of transport at a low cost and with high accuracy. Koutras et al. (2021) developed a comprehensive biomechanical vibration model of the torso, including soft tissue, ribcage and spine under typical loading conditions of scoliosis braces.

In this work, the dynamic behaviour of a whole human body in the driving vehicle is modelled using a biomechanical vibration model, analytically and numerically, to estimate the body's apparent mass and transmissibility and understand the effects of low-frequency vibration on human health, comfort, and performance. The analytical model is useful if quick calculations are required for simple systems to predict the human body's vibration without extensive calculations. Furthermore, analytical models are useful in that they may include a large portion of the relevant human body physics while at the same time having relatively short solution times. The analytical solution model is presented by driving the general equation of motion for the dynamic human body model and calculating the natural frequencies for each human part. In addition, the numerical technique uses the finite element method is also presented to calculate natural frequency and the dynamic response performed for the human body. When using the finite element method, the numerical biomechanical vibration models can study the effect of many properties and operating parameters and, therefore, much more computationally intensive, leading to longer solution times (Desai et al., 2018). However, these disadvantages are typically outweighed by the benefit of assessing the influence of more design parameters and their associated physical processes with high sensitivity and accuracy. Finally, the natural frequency results from both models, i.e. analytical and numerical, are compared with the available published experimental results.

\section{ANALYTICAL TECHNIQUE}

\section{Biomechanical Vibration Model}

In the case of the driving vehicle, the dynamic response of the human body can be obtained using the model shown in Figure 1. This biomedical model can be employed to simulate and design vibration isolators. In this work, the human body is positioned in a healthy posture and looks straight ahead without sideway movements with a relaxed upper body upright state. Also, it assumes that the hands were put on the lap and the foot is on the footplate. The biomechanical model of this human body consists of body parts such as the head, torso, thighs, pelvis, viscera, and legs, as shown in Figure 1. 


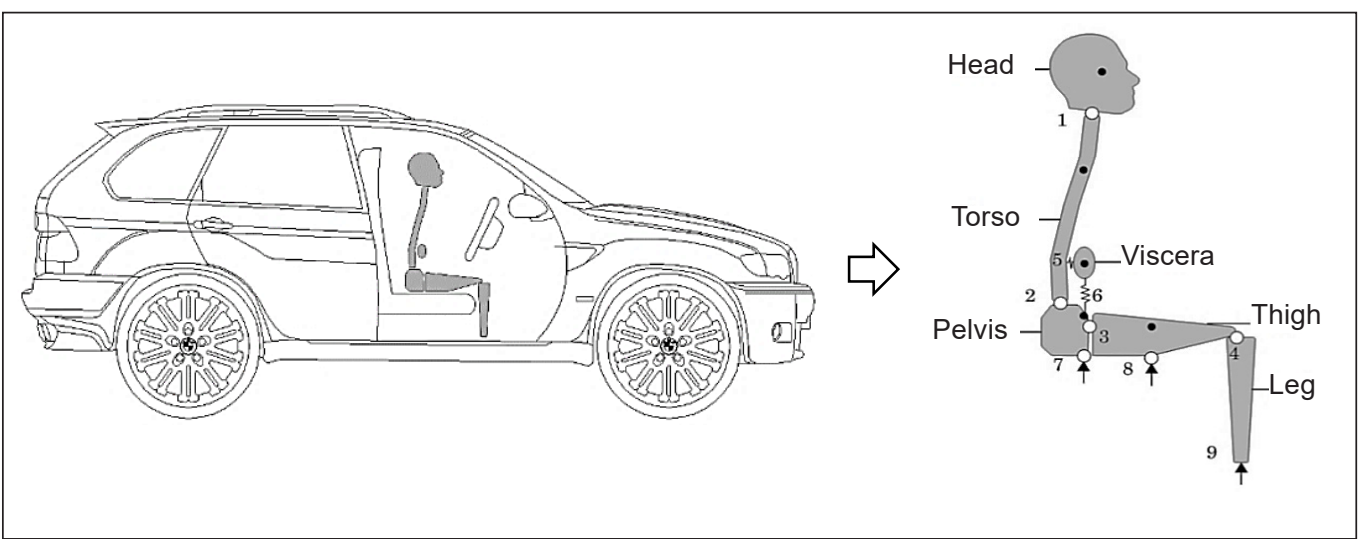

Figure 1. The geometry of the computational biomechanical model showing different parts of the human body and connections between them

The numbers refer to joints between two parts (white circles), and the centre of mass of each part of the body (black circles) and the vibration excitation locations (arrows) are also shown. Figure 1 shows that the primary vibration source in the desired biomechanical model is a seat in a vehicle found not modelled explicitly. Instead, a motion of the base node is used in the geometry of the computational model. Hence, the input excitation at three different locations in the vertical direction was employed. All the body parts in this model are treated as lumped masses, defined as rigid bodies. The viscera in this model are not allowed to turn, and the legs are also not allowed to translate as well rotate. The biomechanical model connections of the body parts are represented by translational and rotational springs and dashpot damper, which can predict the relative motion between the body segments. These types of joints are modelled using the elastic version of a fixed link. Once the flexible version of joints is used, the torsional stiffness and linear damper coefficient are assigned at the joints between the body parts will be provided. Therefore, the human biomechanical models for mechanical properties, motion description in an arbitrary position, and properties for inertia and geometry for a biomechanical model can be simulated in Figures 2 to 4, respectively (Griffin, 1990).

In the assumption of small deformation for individual vertebrae, the strain represents the rigid body rotation. Besides, the prediction of the spinal forces did not define; then, the human biomechanical joint modelling did not include the disks and the vertebrae. Also, it assumed no movements translational between the rigid bodies and its bodies connected by joints as pin joints. The linear spring and damper were considering modelling the shear and axial deformation for the thigh tissue and pelvis. The interface seat occupant frication force and the relative motion between the human body and the seat are neglected (Griffin, 1990). The human body parts directly in touch with the vibrating seat are legs, thighs, and pelvis. The connections between the human body and the vibrating seat are modelled using 
a fixed joint. It is required to model the cushioning effect that the elastic behaviours on these joints are included everywhere of the seat. Also, the legs in this model are connected to the seat with the rigid version of fixed joint, and thus, it does not need to insert joint elasticity parameters. Table 1 presents the mechanical properties values such as mass and moment of inertia of each human body part about its centre of mass (Zheng et al., 2011), while the values of stiffness, damping coefficients, translational and rotational, for all the connections in the biomechanical model are listed in Table 2 (Zheng et al., 2011).

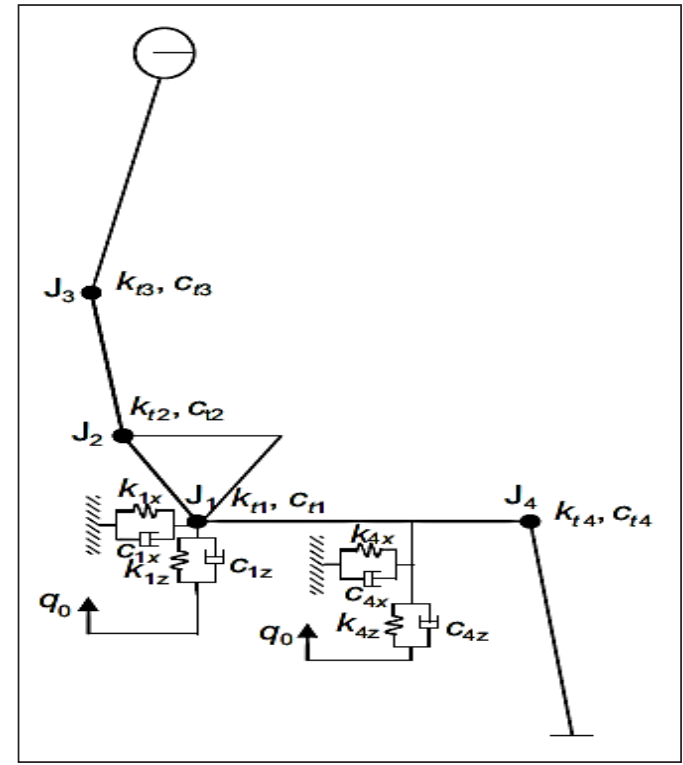

Figure 2. Mechanical properties modelling of the human biomechanical model

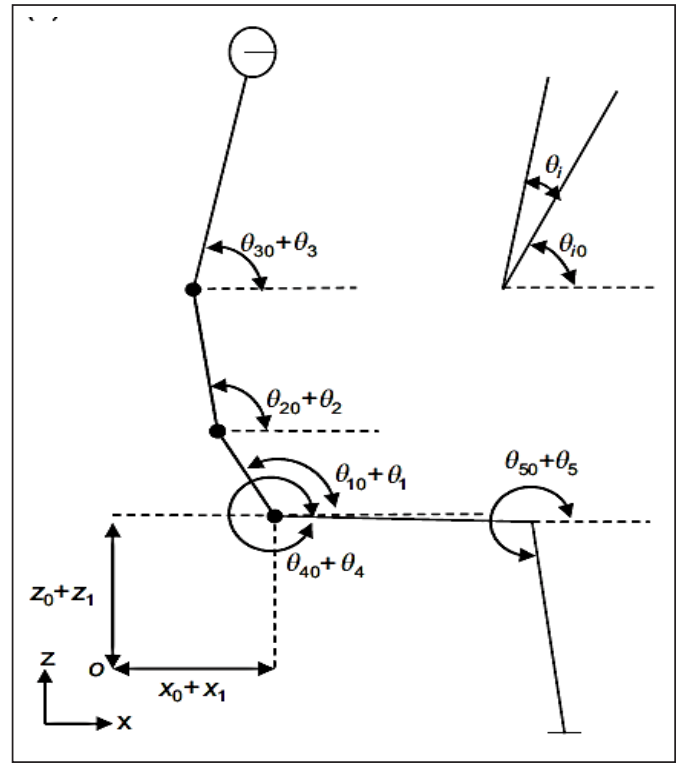

Figure 3. Description for motion in an arbitrary position of the biomechanical model

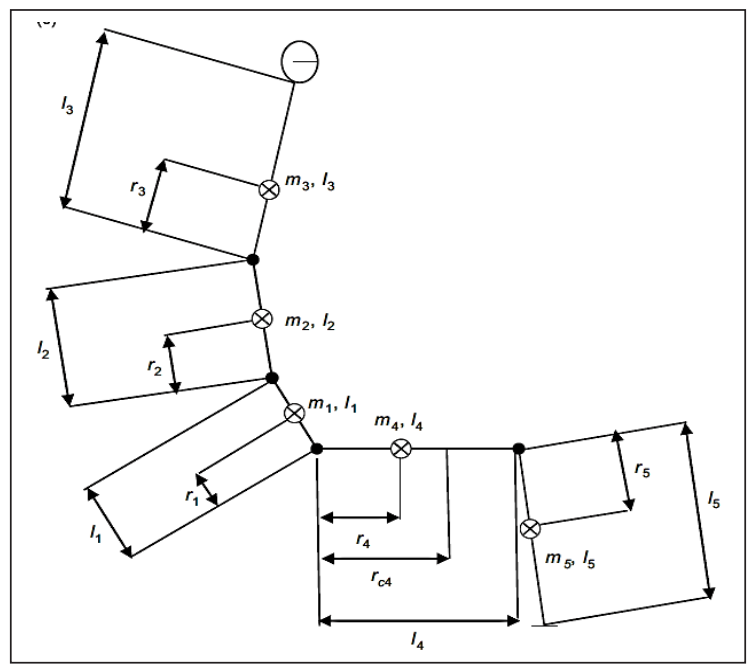

Figure 4. Properties for inertia and geometry for the biomechanical model 
Table 1

Mass and moment of inertia of different human body parts

\begin{tabular}{cccc}
\hline & Mass $(\mathrm{kg})$ & \multicolumn{2}{c}{ Mass moment of inertia $\left(\mathrm{kg} \cdot \mathrm{m}^{2}\right)$} \\
\hline $\mathrm{m}_{1}$ & 8.92 & $1_{1}$ & 0.0249 \\
$\mathrm{~m}_{2}$ & 10.97 & $1_{2}$ & 0.1186 \\
$\mathrm{~m}_{3}$ & 22.64 & $1_{3}$ & 1.28 \\
$\mathrm{~m}_{4}$ & 17.56 & $1_{4}$ & 0.45 \\
$\mathrm{~m}_{5}$ & 8.51 & $1_{5}$ & 0.13 \\
\hline
\end{tabular}

Table 2

Joint elasticity details for human body parts of the biomechanical model

\begin{tabular}{llcccc}
\hline Joint & Fixed joint & $\begin{array}{c}\text { Translational } \\
\text { stiffness }(\mathrm{kN} / \mathrm{m})\end{array}$ & $\begin{array}{c}\text { Translational } \\
\text { damping }(\mathrm{kN} . \mathrm{s} / \mathrm{m})\end{array}$ & $\begin{array}{c}\text { Rotational stiffness } \\
(\mathrm{kN} . \mathrm{m} / \mathrm{Rad})\end{array}$ & $\begin{array}{c}\text { Rotational damping } \\
(\mathrm{kN} . \mathrm{m} . \mathrm{s} / \mathrm{Rad})\end{array}$ \\
\hline 1 & Head-torso & $113.7,113.7$ & $0.066,0.066$ & 0.915 & 0.340 \\
2 & Torso-pelvis & $0.299,0.299$ & $1.79,1.79$ & 0.328 & 0.724 \\
3 & Pelvis-thigh & $6.40,6.40$ & $0.061,0.061$ & 0.162 & 0.030 \\
4 & Thigh-leg & $23.55,23.55$ & $0.154,0.154$ & 0.220 & 0.104 \\
5 & Viscera-torso & $1.93,0$ & $0.079,0$ & 0 & 0 \\
6 & Viscera-pelvis & $0,18.37$ & $0,0.197$ & 0 & 0 \\
7 & Seat-pelvis & $0.905,121.3$ & $0.015,0.047$ & 0 & 0 \\
8 & Seat-thigh & $0.614,16.71$ & $0.014,8.01$ & 0 & 0 \\
9 & Seat-leg & - & - & - & - \\
\hline
\end{tabular}

\section{Biomechanical Vibration Analysis}

The general equation of motion for the biomechanical model for the human body can be evaluated by assuming multi translational and rotational displacement on each mass. Therefore, the general coordinates can be considered small displacement with various time representations and be listed as Equation 1.

$$
\{\delta\}=\left\{\begin{array}{lllllll}
x_{1} & z_{1} & \theta_{1} & \theta_{2} & \theta_{3} & \theta_{4} & \theta_{5}
\end{array}\right\}^{t}
$$

Therefore, by using the energy Lagrange technique can be derived the general equations of motion for the biomechanical model for vibration behaviour of the human body, for assuming small and linear vibration response for each human body part, as Equations 2-4,

$$
\mathrm{T}=\left(\begin{array}{l}
\frac{1}{2} \mathrm{~m}_{1}\left(\dot{\mathrm{x}}_{1}^{2}+\left(\dot{\mathrm{z}}_{1}+\mathrm{r}_{1} \dot{\theta}_{1}\right)^{2}\right)+\frac{1}{2} \mathrm{~m}_{2}\left(\dot{\mathrm{x}}_{1}^{2}+\left(\dot{\mathrm{z}}_{1}+\mathrm{l}_{1} \dot{\theta}_{1}+\mathrm{r}_{2} \dot{\theta}_{2}\right)^{2}\right)+ \\
\frac{1}{2} \mathrm{~m}_{3}\left(\dot{\mathrm{x}}_{1}^{2}+\left(\dot{\mathrm{z}}_{1}+\mathrm{l}_{1} \dot{\theta}_{1}+\mathrm{l}_{2} \dot{\theta}_{2}+\mathrm{r}_{3} \dot{\theta}_{3}\right)^{2}\right)+\frac{1}{2} \mathrm{~m}_{4}\left(\dot{\mathrm{x}}_{1}^{2}+\left(\dot{\mathrm{z}}_{1}+\mathrm{r}_{4} \dot{\theta}_{4}\right)^{2}\right)+ \\
\frac{1}{2} \mathrm{~m}_{5}\left(\dot{\mathrm{x}}_{1}^{2}+\left(\dot{\mathrm{z}}_{1}+\mathrm{l}_{4} \dot{\theta}_{4}+\mathrm{r}_{5} \dot{\theta}_{5}\right)^{5}\right)+\frac{1}{2} \mathrm{I}_{1} \dot{\theta}_{1}^{2}+\frac{1}{2} \mathrm{I}_{2} \dot{\theta}_{2}^{2}+\frac{1}{2} \mathrm{I}_{3} \dot{\theta}_{3}^{2}+\frac{1}{2} \mathrm{I}_{4} \dot{\theta}_{4}^{2}+\frac{1}{2} \mathrm{I}_{5} \dot{\theta}_{5}^{2}
\end{array}\right)
$$




$$
\begin{aligned}
& \mathrm{U}=\left(\begin{array}{l}
\frac{1}{2}\left(\mathrm{k}_{1 \mathrm{x}}+\mathrm{k}_{4 \mathrm{x}}\right) \mathrm{x}_{1}^{2}+\frac{1}{2} \mathrm{k}_{1 \mathrm{z}}\left(\mathrm{z}_{1}-\mathrm{q}_{\mathrm{o}}\right)^{2}+\frac{1}{2} \mathrm{k}_{4 \mathrm{z}}\left(\mathrm{z}_{1}+\mathrm{r}_{\mathrm{c} 4} \theta_{4}-\mathrm{q}_{\mathrm{o}}\right)^{2}+\frac{1}{2} \mathrm{k}_{\mathrm{t} 1}\left(\theta_{1}+\theta_{4}\right)^{2}+ \\
\frac{1}{2} \mathrm{k}_{\mathrm{t} 2}\left(\theta_{1}-\theta_{2}\right)^{2}+\frac{1}{2} \mathrm{k}_{\mathrm{t} 3}\left(\theta_{2}-\theta_{3}\right)^{2}+\frac{1}{2} \mathrm{k}_{\mathrm{t} 4}\left(\theta_{4}+\theta_{5}\right)^{2}
\end{array}\right) \\
& \mathrm{D}=\left(\begin{array}{l}
\frac{1}{2}\left(\mathrm{c}_{1 \mathrm{x}}+\mathrm{c}_{4 \mathrm{x}}\right) \dot{\mathrm{x}}_{1}^{2}+\frac{1}{2} \mathrm{c}_{1 \mathrm{z}}\left(\dot{\mathrm{z}}_{1}-\dot{\mathrm{q}}_{\mathrm{o}}\right)^{2}+\frac{1}{2} \mathrm{c}_{4 \mathrm{z}}\left(\dot{\mathrm{z}}_{1}+\mathrm{r}_{\mathrm{c} 4} \dot{\theta}_{4}-\dot{\mathrm{q}}_{\mathrm{o}}\right)^{2}+\frac{1}{2} \mathrm{c}_{\mathrm{t} 1}\left(\dot{\theta}_{1}+\dot{\theta}_{4}\right)^{2}+ \\
\frac{1}{2} \mathrm{c}_{\mathrm{t} 2}\left(\dot{\theta}_{1}-\dot{\theta}_{2}\right)^{2}+\frac{1}{2} \mathrm{c}_{\mathrm{t} 3}\left(\dot{\theta}_{2}-\dot{\theta}_{3}\right)^{2}+\frac{1}{2} \mathrm{c}_{\mathrm{t} 4}\left(\dot{\theta}_{4}+\dot{\theta}_{5}\right)^{2}
\end{array}\right)
\end{aligned}
$$

Where, $1_{1}$ to $1_{5}$ and $r_{1}$ to $r_{5}$ are presenting in Table 3 , the translational and rotational stiffness is shown in Table 4, and the translational and rotational damping coefficient are shown in Table 5, and $\mathrm{r}_{4}$ used about (14.8) (Zheng et al., 2011).

Then, by using Lagrange's equations to derive the general equations of motion for multi-degree of freedom, by using Equation 5,

$$
\frac{\mathrm{d}}{\mathrm{dt}}\left(\frac{\partial \mathrm{T}}{\partial \dot{\delta}_{\mathrm{i}}}\right)-\left(\frac{\partial \mathrm{T}}{\partial \delta_{\mathrm{i}}}\right)+\left(\frac{\partial \mathrm{D}}{\partial \dot{\delta}_{\mathrm{i}}}\right)+\left(\frac{\partial \mathrm{U}}{\partial \delta_{\mathrm{i}}}\right)=\mathrm{F}_{\mathrm{i}}
$$

Where, i number of degree of freedom, and $\mathrm{F}_{\mathrm{i}}$ applied load.

Table 3

Dimensions for human body parts

\begin{tabular}{cccc}
\hline \multicolumn{2}{c}{ Part length $(\mathrm{cm})$} & \multicolumn{2}{c}{ Mass centre $(\mathrm{cm})$} \\
\hline $1_{1}$ & 13.77 & $\mathrm{r}_{1}$ & 9.78 \\
$1_{2}$ & 29.65 & $\mathrm{r}_{2}$ & 16.26 \\
$1_{3}$ & 40.27 & $\mathrm{r}_{3}$ & 20.14 \\
$1_{4}$ & 34.6 & $\mathrm{r}_{4}$ & 14.8 \\
$1_{5}$ & 36.33 & $\mathrm{r}_{5}$ & 15.49 \\
\hline
\end{tabular}

Table 4

Translational and rotational stiffness for biomechanical human body parts

\begin{tabular}{cccc}
\hline Translational stiffness $(\mathrm{kN} / \mathrm{m})$ & Rotational stiffness $(\mathrm{kN} . \mathrm{m} / \mathrm{rad})$ \\
\hline $\mathrm{k}_{\mathrm{lx}}$ & 0.905 & $\mathrm{k}_{\mathrm{t} 1}$ & 0.162 \\
$\mathrm{k}_{4 \mathrm{x}}$ & 0.614 & $\mathrm{k}_{\mathrm{t} 2}$ & 0.328 \\
$\mathrm{k}_{\mathrm{kz}}$ & 121.3 & $\mathrm{k}_{\mathrm{t} 3}$ & 0.915 \\
$\mathrm{k}_{4 \mathrm{z}}$ & 16.71 & $\mathrm{k}_{\mathrm{t} 4}$ & 0.22 \\
\hline
\end{tabular}

Table 5

Translational and rotational damping coefficient for the human body

\begin{tabular}{cccc}
\hline Translational stiffness $(\mathrm{kN} . \mathrm{s} / \mathrm{m})$ & Rotational stiffness & (kN.m.s/rad) \\
\hline $\mathrm{c}_{1 \mathrm{x}}$ & 0.015 & $\mathrm{c}_{\mathrm{t} 1}$ & 0.03 \\
$\mathrm{c}_{4 \mathrm{x}}$ & 0.014 & $\mathrm{c}_{\mathrm{t} 2}$ & 0.724 \\
$\mathrm{c}_{1 \mathrm{z}}$ & 0.047 & $\mathrm{c}_{\mathrm{t} 3}$ & 0.34 \\
$\mathrm{c}_{4 \mathrm{z}}$ & 8.01 & $\mathrm{c}_{\mathrm{t} 4}$ & 0.104 \\
\hline
\end{tabular}


Then, by using Equations 2-4, can be evaluated using the general equation of motion of the system, as Equations 6-12,

For, $\mathrm{i}=1, \delta_{1}=\mathrm{x}_{1}$,

$$
\begin{aligned}
& \frac{\mathrm{d}}{\mathrm{dt}}\left(\frac{\partial \mathrm{T}}{\partial \dot{\mathrm{x}}_{1}}\right)=\left(\left(\mathrm{m}_{1}+\mathrm{m}_{2}+\mathrm{m}_{3}+\mathrm{m}_{4}+\mathrm{m}_{5}\right) \ddot{\mathrm{x}}_{1}\right),\left(\frac{\partial \mathrm{T}}{\partial \mathrm{x}_{1}}\right)=0 \\
& \left(\frac{\partial \mathrm{D}}{\partial \dot{\mathrm{x}}_{1}}\right)=\left(\left(\mathrm{c}_{1 \mathrm{x}}+\mathrm{c}_{4 \mathrm{x}}\right) \dot{\mathrm{x}}_{1}\right),\left(\frac{\partial \mathrm{U}}{\partial \mathrm{x}_{1}}\right)=\left(\left(\mathrm{k}_{1 \mathrm{x}}+\mathrm{k}_{4 \mathrm{x}}\right) \mathrm{x}_{1}\right)
\end{aligned}
$$

Then,

$$
\left(\mathrm{m}_{1}+\mathrm{m}_{2}+\mathrm{m}_{3}+\mathrm{m}_{4}+\mathrm{m}_{5}\right) \ddot{\mathrm{x}}_{1}+\left(\mathrm{c}_{1 \mathrm{x}}+\mathrm{c}_{4 \mathrm{x}}\right) \dot{\mathrm{x}}_{1}+\left(\mathrm{k}_{1 \mathrm{x}}+\mathrm{k}_{4 \mathrm{x}}\right) \mathrm{x}_{1}=0
$$

For, $\mathrm{i}=2, \delta_{2}=\mathrm{z}_{1}$,

$$
\begin{aligned}
& \frac{\mathrm{d}}{\mathrm{dt}}\left(\frac{\partial \mathrm{T}}{\partial \dot{\mathrm{z}}_{1}}\right)=\left(\begin{array}{c}
\left(\mathrm{m}_{1}+\mathrm{m}_{2}+\mathrm{m}_{3}+\mathrm{m}_{4}+\mathrm{m}_{5}\right) \ddot{\mathrm{z}}_{1}+\left(\mathrm{m}_{1} \mathrm{r}_{1}+\mathrm{m}_{2} \mathrm{l}_{1}+\mathrm{m}_{3} \mathrm{l}_{1}\right) \ddot{\theta}_{1}+ \\
\left(\mathrm{m}_{2} \mathrm{r}_{2}+\mathrm{m}_{3} \mathrm{l}_{2}\right) \ddot{\theta}_{2}+\left(\mathrm{m}_{3} \mathrm{r}_{3}\right) \ddot{\theta}_{3}+\left(\mathrm{m}_{4} \mathrm{r}_{4}+\mathrm{m}_{5} \mathrm{l}_{4}\right) \ddot{\theta}_{4}+\left(\mathrm{m}_{5} \mathrm{r}_{5}\right) \ddot{\theta}_{5}
\end{array}\right) \\
& \left(\frac{\partial \mathrm{T}}{\partial \mathrm{z}_{1}}\right)=0, \\
& \left(\frac{\partial \mathrm{D}}{\partial \dot{\mathrm{z}}_{1}}\right)=\left(\left(\mathrm{c}_{1 \mathrm{z}}+\mathrm{c}_{4 \mathrm{z}}\right) \dot{\mathrm{z}}_{1}+\left(\mathrm{c}_{4 \mathrm{z}} \mathrm{r}_{\mathrm{c} 4}\right) \dot{\theta}_{4}-\left(\mathrm{c}_{1 \mathrm{z}}+\mathrm{c}_{4 \mathrm{z}}\right) \dot{\mathrm{q}}_{\mathrm{o}}\right),\left(\frac{\partial \mathrm{U}}{\partial \mathrm{z}_{1}}\right)= \\
& \left(\left(\mathrm{k}_{1 \mathrm{z}}+\mathrm{k}_{4 \mathrm{z}}\right) \mathrm{z}_{1}+\left(\mathrm{k}_{4 \mathrm{z}} \mathrm{r}_{\mathrm{c} 4}\right) \theta_{4}-\left(\mathrm{k}_{1 \mathrm{z}}+\mathrm{k}_{4 \mathrm{z}}\right) \mathrm{q}_{\mathrm{o}}\right)
\end{aligned}
$$

Then,

$$
\left(\begin{array}{l}
\left(m_{1}+m_{2}+m_{3}+m_{4}+m_{5}\right) \ddot{z}_{1}+\left(m_{1} r_{1}+m_{2} l_{1}+m_{3} l_{1}\right) \ddot{\theta}_{1}+ \\
\left(m_{2} r_{2}+m_{3} l_{2}\right) \ddot{\theta}_{2}+\left(m_{3} r_{3}\right) \ddot{\theta}_{3}+\left(m_{4} r_{4}+m_{5} l_{4}\right) \ddot{\theta}_{4}+\left(m_{5} r_{5}\right) \ddot{\theta}_{5} \\
+\left(c_{1 z}+c_{4 z}\right) \dot{z}_{1}+\left(c_{4 z} r_{c 4}\right) \dot{\theta}_{4}+\left(k_{1 z}+k_{4 z}\right) z_{1}+\left(k_{4 z} r_{c 4}\right) \theta_{4}
\end{array}\right)=\left(\begin{array}{l}
\left(k_{1 z}+k_{4 z}\right) q_{o}+ \\
\left(c_{1 z}+c_{4 z}\right) \dot{q}_{o}
\end{array}\right)
$$

For, $\mathrm{i}=3, \delta_{3}=\theta_{1}$,

$$
\begin{aligned}
& \frac{\mathrm{d}}{\mathrm{dt}}\left(\frac{\partial \mathrm{T}}{\partial \dot{\theta}_{1}}\right)=\left(\begin{array}{c}
\left(\mathrm{m}_{1} \mathrm{r}_{1}+\mathrm{m}_{2} \mathrm{l}_{1}+\mathrm{m}_{3} \mathrm{l}_{1}\right) \ddot{\mathrm{z}}_{1}+\left(\mathrm{m}_{1} \mathrm{r}_{1}^{2}+\mathrm{m}_{2} \mathrm{l}_{1}^{2}+\mathrm{m}_{3} \mathrm{l}_{1}^{2}+\mathrm{I}_{1}\right) \ddot{\theta}_{1} \\
+\left(\mathrm{m}_{2} \mathrm{r}_{2} \mathrm{l}_{1}+\mathrm{m}_{3} \mathrm{l}_{2} \mathrm{l}_{1}\right) \ddot{\theta}_{2}+\left(\mathrm{m}_{3} \mathrm{r}_{3} \mathrm{l}_{1}\right) \ddot{\theta}_{3}
\end{array}\right) \\
& \left(\frac{\partial \mathrm{T}}{\partial \theta_{1}}\right)=0,\left(\frac{\partial \mathrm{D}}{\partial \dot{\theta}_{1}}\right)=\left(\left(\mathrm{c}_{\mathrm{t} 1}+\mathrm{c}_{\mathrm{t} 2}\right) \dot{\theta}_{1}-\left(\mathrm{c}_{\mathrm{t} 2}\right) \dot{\theta}_{2}+\left(\mathrm{c}_{\mathrm{t} 1}\right) \dot{\theta}_{4}\right)\left(\frac{\partial \mathrm{U}}{\partial \theta_{1}}\right)= \\
& \left(\left(\mathrm{k}_{\mathrm{t} 1}+\mathrm{k}_{\mathrm{t} 2}\right) \theta_{1}-\mathrm{k}_{\mathrm{t} 2} \theta_{2}+\left(\mathrm{k}_{\mathrm{t} 1}\right) \theta_{4}\right)
\end{aligned}
$$

Then,

$$
\left(\begin{array}{l}
\left(m_{1} \mathrm{r}_{1}+\mathrm{m}_{2} \mathrm{l}_{1}+\mathrm{m}_{3} \mathrm{l}_{1}\right) \ddot{\mathrm{z}}_{1}+\left(\mathrm{m}_{1} \mathrm{r}_{1}^{2}+\mathrm{m}_{2} \mathrm{l}_{1}^{2}+\mathrm{m}_{3} \mathrm{l}_{1}^{2}+\mathrm{I}_{1}\right) \ddot{\theta}_{1}+\left(\mathrm{m}_{2} \mathrm{r}_{2} \mathrm{l}_{1}+\mathrm{m}_{3} \mathrm{l}_{2} \mathrm{l}_{1}\right) \ddot{\theta}_{2}+ \\
\left(\mathrm{m}_{3} \mathrm{r}_{3} \mathrm{l}_{1}\right) \ddot{\theta}_{3}+\left(\mathrm{c}_{\mathrm{t} 1}+\mathrm{c}_{\mathrm{t} 2}\right) \dot{\theta}_{1}-\left(\mathrm{c}_{\mathrm{t} 2}\right) \dot{\theta}_{2}+\left(\mathrm{c}_{\mathrm{t} 1}\right) \dot{\theta}_{4}+\left(\mathrm{k}_{\mathrm{t} 1}+\mathrm{k}_{\mathrm{t} 2}\right) \theta_{1}-\mathrm{k}_{\mathrm{t} 2} \theta_{2}+\left(\mathrm{k}_{\mathrm{t} 1}\right) \theta_{4}
\end{array}\right)=0
$$

For, $\mathrm{i}=4, \delta_{4}=\theta_{2}$, 


$$
\begin{aligned}
& \frac{\mathrm{d}}{\mathrm{dt}}\left(\frac{\partial \mathrm{T}}{\partial \dot{\theta}_{2}}\right)=\left(\left(\mathrm{m}_{2} \mathrm{r}_{2}+\mathrm{m}_{3} \mathrm{l}_{2}\right) \ddot{\mathrm{z}}_{1}+\left(\mathrm{m}_{2} \mathrm{l}_{1} \mathrm{r}_{2}+\mathrm{m}_{3} \mathrm{l}_{1} \mathrm{l}_{2}\right) \ddot{\theta}_{1}+\left(\mathrm{m}_{2} \mathrm{r}_{2}^{2}+\mathrm{m}_{3} \mathrm{l}_{2}^{2}+\mathrm{I}_{2}\right) \ddot{\theta}_{2}+\left(\mathrm{m}_{3} \mathrm{r}_{3} \mathrm{l}_{2}\right) \ddot{\theta}_{3}\right) \\
& \left(\frac{\partial \mathrm{T}}{\partial \theta_{2}}\right)=0,\left(\frac{\partial \mathrm{D}}{\partial \dot{\theta}_{2}}\right)=\left(\left(\mathrm{c}_{\mathrm{t} 2}+\mathrm{c}_{\mathrm{t} 3}\right) \dot{\theta}_{2}-\left(\mathrm{c}_{\mathrm{t} 2}\right) \dot{\theta}_{1}-\left(\mathrm{c}_{\mathrm{t} 3}\right) \dot{\theta}_{3}\right),\left(\frac{\partial \mathrm{U}}{\partial \theta_{2}}\right)=\left(\begin{array}{c}
\left(\mathrm{k}_{\mathrm{t} 2}+\mathrm{k}_{\mathrm{t} 3}\right) \theta_{2}- \\
\left(\mathrm{k}_{\mathrm{t} 2}\right) \theta_{1}-\left(\mathrm{k}_{\mathrm{t} 3}\right) \theta_{3}
\end{array}\right)
\end{aligned}
$$

Then,

$$
\left(\begin{array}{l}
\left(m_{2} r_{2}+m_{3} l_{2}\right) \ddot{z}_{1}+\left(m_{2} l_{1} r_{2}+m_{3} l_{1} l_{2}\right) \ddot{\theta}_{1}+\left(m_{2} r_{2}^{2}+m_{3} l_{2}^{2}+I_{2}\right) \ddot{\theta}_{2}+\left(m_{3} r_{3} l_{2}\right) \ddot{\theta}_{3}+ \\
\left(c_{t 2}+c_{t}\right) \dot{\theta}_{2}-\left(c_{t 2}\right) \dot{\theta}_{1}-\left(c_{t 3}\right) \dot{\theta}_{3}+\left(k_{t 2}+k_{t 3}\right) \theta_{2}-\left(k_{t 2}\right) \theta_{1}-\left(k_{t 3}\right) \theta_{3}
\end{array}\right)=0
$$

For, $\mathrm{i}=5, \delta_{5}=\theta_{3}$,

$$
\begin{aligned}
& \frac{\mathrm{d}}{\mathrm{dt}}\left(\frac{\partial \mathrm{T}}{\partial \dot{\theta}_{3}}\right)=\left(\left(\mathrm{m}_{3} \mathrm{r}_{3}\right) \ddot{z}_{1}+\left(\mathrm{m}_{3} \mathrm{l}_{1} \mathrm{r}_{3}\right) \ddot{\theta}_{1}+\left(\mathrm{m}_{3} \mathrm{l}_{2} \mathrm{r}_{3}\right) \ddot{\theta}_{2}+\left(\mathrm{m}_{3} \mathrm{r}_{3}^{2}+\mathrm{I}_{3}\right) \ddot{\theta}_{3}\right) \\
& \left(\frac{\partial \mathrm{T}}{\partial \theta_{3}}\right)=0,\left(\frac{\partial \mathrm{D}}{\partial \dot{\theta}_{3}}\right)=\left(\left(\mathrm{c}_{\mathrm{t} 3}\right) \dot{\theta}_{3}-\left(\mathrm{c}_{\mathrm{t} 3}\right) \dot{\theta}_{2}\right),\left(\frac{\partial \mathrm{U}}{\partial \theta_{3}}\right)=\left(\mathrm{k}_{\mathrm{t} 3} \theta_{3}-\mathrm{k}_{\mathrm{t} 3} \theta_{2}\right)
\end{aligned}
$$

Then,

$$
\left(\begin{array}{c}
\left(m_{3} r_{3}\right) \ddot{z}_{1}+\left(m_{3} l_{1} r_{3}\right) \ddot{\theta}_{1}+\left(m_{3} l_{2} r_{3}\right) \ddot{\theta}_{2}+\left(m_{3} r_{3}^{2}+I_{3}\right) \ddot{\theta}_{3}+\left(c_{t 3}\right) \dot{\theta}_{3}- \\
\left(c_{t 3}\right) \dot{\theta}_{2}+k_{t 3} \theta_{3}-k_{t 3} \theta_{2}
\end{array}\right)=0
$$

For, $\mathrm{i}=6, \delta_{6}=\theta_{4}$,

$$
\begin{aligned}
& \frac{\mathrm{d}}{\mathrm{dt}}\left(\frac{\partial \mathrm{T}}{\partial \dot{\theta}_{4}}\right)=\left(\left(\mathrm{m}_{4} \mathrm{r}_{4}+\mathrm{m}_{5} \mathrm{l}_{4}\right) \ddot{\mathrm{z}}_{1}+\left(\mathrm{m}_{5} \mathrm{r}_{5} \mathrm{l}_{4}\right) \ddot{\theta}_{5}+\left(\mathrm{m}_{4} \mathrm{r}_{4}^{2}+\mathrm{m}_{5} \mathrm{l}_{4}^{2}+\mathrm{I}_{4}\right) \ddot{\theta}_{4}\right) \\
& \left(\frac{\partial \mathrm{T}}{\partial \theta_{4}}\right)=0 \\
& \left(\frac{\partial \mathrm{D}}{\partial \dot{\theta}_{4}}\right)=\left(\left(\mathrm{c}_{4 \mathrm{z}} \mathrm{r}_{\mathrm{c} 4}\right) \dot{\mathrm{z}}_{1}+\left(\mathrm{c}_{\mathrm{t} 1}\right) \dot{\theta}_{1}+\left(\mathrm{c}_{\mathrm{t} 4}\right) \dot{\theta}_{5}+\left(\mathrm{c}_{4 \mathrm{z}} \mathrm{r}_{\mathrm{c} 4}^{2}+\mathrm{c}_{\mathrm{t} 1}+\mathrm{c}_{\mathrm{t} 4}\right) \dot{\theta}_{4}-\left(\mathrm{c}_{4 \mathrm{z}} \mathrm{r}_{\mathrm{c} 4}\right) \dot{\mathrm{q}}_{\mathrm{o}}\right) \\
& \left(\frac{\partial \mathrm{U}}{\partial \theta_{4}}\right)=\left(\left(\mathrm{k}_{4 \mathrm{z}} \mathrm{r}_{\mathrm{c} 4}\right) \mathrm{z}_{1}+\left(\mathrm{k}_{\mathrm{t} 1}\right) \theta_{1}+\mathrm{k}_{\mathrm{t} 4} \theta_{5}+\left(\mathrm{k}_{4 \mathrm{z}} \mathrm{r}_{\mathrm{c} 4}^{2}+\mathrm{k}_{\mathrm{t} 1}+\mathrm{k}_{\mathrm{t} 4}\right) \theta_{4}-\mathrm{k}_{4 \mathrm{z}} \mathrm{r}_{\mathrm{c} 4} \mathrm{q}_{\mathrm{o}}\right)
\end{aligned}
$$

Then,

$$
\left(\begin{array}{c}
\left(\mathrm{m}_{4} \mathrm{r}_{4}+\mathrm{m}_{5} \mathrm{l}_{4}\right) \ddot{\mathrm{z}}_{1}+\left(\mathrm{m}_{5} \mathrm{r}_{5} \mathrm{l}_{4}\right) \ddot{\theta}_{5}+\left(\mathrm{m}_{4} \mathrm{r}_{4}^{2}+\mathrm{m}_{5} \mathrm{l}_{4}^{2}+\mathrm{I}_{4}\right) \ddot{\theta}_{4}+ \\
\left(\mathrm{c}_{4 \mathrm{z}} \mathrm{r}_{\mathrm{c} 4}\right) \dot{\mathrm{z}}_{1}+\left(\mathrm{c}_{\mathrm{t} 1}\right) \dot{\theta}_{1}+\left(\mathrm{c}_{\mathrm{t} 4}\right) \dot{\theta}_{5}+\left(\mathrm{c}_{4 \mathrm{z}} \mathrm{r}_{\mathrm{c} 4}^{2}+\mathrm{c}_{\mathrm{t} 1}+\mathrm{c}_{\mathrm{t} 4}\right) \dot{\theta}_{4}+ \\
\left(\mathrm{k}_{4 \mathrm{z}} \mathrm{r}_{\mathrm{c} 4}\right) \mathrm{z}_{1}+\left(\mathrm{k}_{\mathrm{t} 1}\right) \theta_{1}+\mathrm{k}_{\mathrm{t} 4} \theta_{5}+\left(\mathrm{k}_{4 \mathrm{z}} \mathrm{r}_{\mathrm{c} 4}^{2}+\mathrm{k}_{\mathrm{t} 1}+\mathrm{k}_{\mathrm{t} 4}\right) \theta_{4}
\end{array}\right)=\left(\mathrm{k}_{4 \mathrm{z}} \mathrm{r}_{\mathrm{c} 4} \mathrm{q}_{\mathrm{o}}+\left(\mathrm{c}_{4 \mathrm{z}} \mathrm{r}_{\mathrm{c} 4}\right) \dot{\mathrm{q}}_{\mathrm{o}}\right)
$$

For, $\mathrm{i}=7, \delta_{7}=\theta_{5}$,

$$
\begin{aligned}
& \frac{\mathrm{d}}{\mathrm{dt}}\left(\frac{\partial \mathrm{T}}{\partial \dot{\theta}_{5}}\right)=\left(\left(\mathrm{m}_{5} \mathrm{r}_{5}\right) \ddot{z}_{1}+\left(\mathrm{m}_{5} \mathrm{l}_{4} \mathrm{r}_{5}\right) \ddot{\theta}_{4}+\left(\mathrm{m}_{5} \mathrm{r}_{5}^{2}+\mathrm{I}_{5}\right) \ddot{\theta}_{5}\right) \\
& \left(\frac{\partial \mathrm{T}}{\partial \theta_{5}}\right)=0,\left(\frac{\partial \mathrm{D}}{\partial \dot{\theta}_{5}}\right)=\left(\mathrm{c}_{\mathrm{t} 4} \dot{\theta}_{4}+\mathrm{c}_{\mathrm{t} 4} \dot{\theta}_{5}\right),\left(\frac{\partial \mathrm{U}}{\partial \theta_{5}}\right)=\left(\mathrm{k}_{\mathrm{t} 4} \theta_{4}+\mathrm{k}_{\mathrm{t} 4} \theta_{5}\right)
\end{aligned}
$$


Then,

$$
\left(\left(m_{5} r_{5}\right) \ddot{z}_{1}+\left(m_{5} l_{4} r_{5}\right) \ddot{\theta}_{4}+\left(m_{5} r_{5}^{2}+I_{5}\right) \ddot{\theta}_{5}+c_{t 4} \dot{\theta}_{4}+c_{t 4} \dot{\theta}_{5}+k_{t 4} \theta_{4}+k_{t 4} \theta_{5}\right)=0
$$

Therefore, Equations 6-12, can be rewritten by using matrix form, as Equations 13 and 14,

$$
[\mathrm{m}]\{\ddot{\delta}\}+[\mathrm{c}]\{\dot{\delta}\}+[\mathrm{k}]\{\delta\}=\{\mathrm{F}\}
$$

Where,

$$
[\mathrm{m}]=\left[\begin{array}{ccc}
\mathrm{m}_{11} & \cdots & \mathrm{m}_{17} \\
\vdots & \ddots & \vdots \\
\mathrm{m}_{71} & \cdots & \mathrm{m}_{77}
\end{array}\right]
$$

For,

$$
\begin{aligned}
& \mathrm{m}_{11}=\left(\mathrm{m}_{1}+\mathrm{m}_{2}+\mathrm{m}_{3}+\mathrm{m}_{4}+\mathrm{m}_{5}\right), \mathrm{m}_{12}=\mathrm{m}_{13}=\mathrm{m}_{14}=\mathrm{m}_{15}=\mathrm{m}_{16}=\mathrm{m}_{17}=0 \\
& \mathrm{~m}_{22}=\left(\mathrm{m}_{1}+\mathrm{m}_{2}+\mathrm{m}_{3}+\mathrm{m}_{4}+\mathrm{m}_{5}\right), \mathrm{m}_{23}=\left(\mathrm{m}_{1} \mathrm{r}_{1}+\mathrm{m}_{2} \mathrm{l}_{1}+\mathrm{m}_{3} \mathrm{l}_{1}\right), \mathrm{m}_{24}=\left(\mathrm{m}_{2} \mathrm{r}_{2}+\mathrm{m}_{3} \mathrm{l}_{2}\right) \\
& \mathrm{m}_{25}=\left(\mathrm{m}_{3} \mathrm{r}_{3}\right), \mathrm{m}_{26}=\left(\mathrm{m}_{4} \mathrm{r}_{4}+\mathrm{m}_{5} \mathrm{l}_{4}\right), \mathrm{m}_{27}=\left(\mathrm{m}_{5} \mathrm{r}_{5}\right), \mathrm{m}_{33}=\left(\mathrm{m}_{1} \mathrm{r}_{1}^{2}+\mathrm{m}_{2} \mathrm{l}_{1}^{2}+\mathrm{m}_{3} \mathrm{l}_{1}^{2}+\mathrm{I}_{1}\right) \\
& \mathrm{m}_{34}=\left(\mathrm{m}_{2} \mathrm{r}_{2} \mathrm{l}_{1}+\mathrm{m}_{3} \mathrm{l}_{2} \mathrm{l}_{1}\right), \mathrm{m}_{35}=\left(\mathrm{m}_{3} \mathrm{r}_{3} \mathrm{l}_{1}\right), \mathrm{m}_{36}=\mathrm{m}_{37}=0, \mathrm{~m}_{44}=\left(\mathrm{m}_{2} \mathrm{r}_{2}^{2}+\mathrm{m}_{3} \mathrm{l}_{2}^{2}+\mathrm{I}_{2}\right) \\
& \mathrm{m}_{45}=\left(\mathrm{m}_{3} \mathrm{r}_{3} \mathrm{l}_{2}\right), \mathrm{m}_{46}=\mathrm{m}_{47}=0, \mathrm{~m}_{55}=\left(\mathrm{m}_{3} \mathrm{r}_{3}^{2}+\mathrm{I}_{3}\right), \mathrm{m}_{56}=\mathrm{m}_{57}=0, \\
& \mathrm{~m}_{66}=\left(\mathrm{m}_{4} \mathrm{r}_{4}^{2}+\mathrm{m}_{5} \mathrm{l}_{4}^{2}+\mathrm{I}_{4}\right) \\
& \mathrm{m}_{67}=\left(\mathrm{m}_{5} \mathrm{r}_{5} \mathrm{l}_{4}\right), \mathrm{m}_{77}=\left(\mathrm{m}_{5} \mathrm{r}_{5}^{2}+\mathrm{I}_{5}\right)
\end{aligned}
$$

And,

$$
[c]=\left[\begin{array}{ccc}
c_{11} & \cdots & c_{17} \\
\vdots & \ddots & \vdots \\
c_{71} & \cdots & c_{77}
\end{array}\right]
$$

For,

$$
\begin{aligned}
& c_{11}=\left(c_{1 x}+c_{4 x}\right), c_{12}=c_{13}=c_{14}=c_{15}=c_{16}=c_{17}=0, c_{22}=\left(c_{1 z}+c_{4 z}\right), c_{26}=\left(c_{4 z} r_{c 4}\right) \\
& c_{23}=c_{24}=c_{25}=c_{27}=0, c_{33}=\left(c_{t 1}+c_{t 2}\right), c_{34}=-\left(c_{t 2}\right), c_{36}=\left(c_{t 1}\right), c_{35}=c_{37}=0, c_{44}= \\
& \left(c_{t 2}+c_{t 3}\right), c_{45}=-\left(c_{t 3}\right), c_{46}=c_{47}=0, c_{55}=\left(c_{t 3}\right), c_{56}=c_{57}=0, \\
& c_{66}=\left(c_{4 z} r_{c 4}^{2}+c_{t 1}+c_{t 4}\right), c_{67}=\left(c_{t 4}\right), c_{77}=\left(c_{t 4}\right)
\end{aligned}
$$

Also,

$$
[\mathrm{k}]=\left[\begin{array}{ccc}
\mathrm{k}_{11} & \cdots & \mathrm{k}_{17} \\
\vdots & \ddots & \vdots \\
\mathrm{k}_{71} & \cdots & \mathrm{k}_{77}
\end{array}\right]
$$

For,

$$
\begin{aligned}
& \mathrm{k}_{11}=\left(\mathrm{k}_{1 \mathrm{x}}+\mathrm{k}_{4 \mathrm{x}}\right), \mathrm{k}_{12}=\mathrm{k}_{13}=\mathrm{k}_{14}=\mathrm{k}_{15}=\mathrm{k}_{16}=\mathrm{k}_{17}=0, \mathrm{k}_{22}=\left(\mathrm{k}_{1 \mathrm{z}}+\mathrm{k}_{4 \mathrm{z}}\right), \\
& \mathrm{k}_{26}=\left(\mathrm{k}_{4 \mathrm{z}} \mathrm{r}_{\mathrm{c} 4}\right)
\end{aligned}
$$




$$
\begin{aligned}
& \mathrm{k}_{23}=\mathrm{k}_{24}=\mathrm{k}_{25}=\mathrm{k}_{27}=0, \mathrm{k}_{33}=\left(\mathrm{k}_{\mathrm{t} 1}+\mathrm{k}_{\mathrm{t} 2}\right), \mathrm{k}_{34}=-\left(\mathrm{k}_{\mathrm{t} 2}\right), \mathrm{k}_{36}=\left(\mathrm{k}_{\mathrm{t} 1}\right), \mathrm{k}_{35}= \\
& \mathrm{k}_{37}=0, \mathrm{k}_{44}=\left(\mathrm{k}_{\mathrm{t} 2}+\mathrm{k}_{\mathrm{t} 3}\right), \mathrm{k}_{45}=-\left(\mathrm{k}_{\mathrm{t} 3}\right), \mathrm{k}_{46}=\mathrm{k}_{47}=0, \mathrm{k}_{55}=\mathrm{k}_{\mathrm{t} 3}, \mathrm{k}_{56}=\mathrm{k}_{57}=0, \\
& \mathrm{k}_{66}=\left(\mathrm{k}_{4 \mathrm{z}} \mathrm{r}_{\mathrm{c} 4}^{2}+\mathrm{k}_{\mathrm{t} 1}+\mathrm{k}_{\mathrm{t} 4}\right), \mathrm{k}_{67}=\left(\mathrm{k}_{\mathrm{t} 4}\right), \mathrm{k}_{77}=\left(\mathrm{k}_{\mathrm{t} 4}\right)
\end{aligned}
$$

And,

$$
\begin{gathered}
\{\ddot{\delta}\}=\left\{\begin{array}{c}
\ddot{\mathrm{x}}_{1} \\
\ddot{\mathrm{z}}_{1} \\
\ddot{\theta}_{1} \\
\ddot{\theta}_{2} \\
\ddot{\theta}_{3} \\
\ddot{\theta}_{4} \\
\ddot{\theta}_{5}
\end{array}\right\},\{\dot{\delta}\}=\left\{\begin{array}{c}
\dot{\mathrm{x}}_{1} \\
\dot{\mathrm{z}}_{1} \\
\dot{\theta}_{1} \\
\dot{\theta}_{2} \\
\dot{\theta}_{3} \\
\dot{\theta}_{4} \\
\dot{\theta}_{5}
\end{array}\right\},\{\delta\}=\left\{\begin{array}{c}
\mathrm{x}_{1} \\
\mathrm{z}_{1} \\
\theta_{1} \\
\theta_{2} \\
\theta_{3} \\
\theta_{4} \\
\theta_{5}
\end{array}\right\} \\
0 \mathrm{~F}\}=\left\{\begin{array}{c}
\left(\mathrm{k}_{1 \mathrm{z}}+\mathrm{k}_{4 \mathrm{z}}\right) \mathrm{q}_{\mathrm{o}}+\left(\mathrm{c}_{1 \mathrm{z}}+\mathrm{c}_{4 \mathrm{z}}\right) \dot{\mathrm{q}}_{\mathrm{o}} \\
0 \\
0 \\
0 \\
\left(\left(\mathrm{k}_{4 \mathrm{z}} \mathrm{r}_{\mathrm{c} 4}\right) \mathrm{q}_{\mathrm{o}}+\left(\mathrm{c}_{4 \mathrm{z}} \mathrm{r}_{\mathrm{c} 4}\right) \dot{\mathrm{q}}_{\mathrm{o}}\right) \\
0
\end{array}\right\}
\end{gathered}
$$

Where, $\mathrm{q}_{\mathrm{o}}$ is the load-displacement, which can be assumed with a frequency range from 0.3 to $20 \mathrm{~Hz}$ (Zheng et al., 2011).

The mathematical modelling for vibration, translational and rotational motion for the biomechanical human body has been discussed to generate the eigenvalue problem. This analysis included solving for the equation of motion for, Equation 13 (Al-Waily, 2005; Rao, 2007; Rao, 2018; Abbas et al., 2019) to calculate natural frequencies for the human body, as Equation 15,

$$
\left|[\mathrm{k}]-\omega^{2}[\mathrm{~m}]\right|[\mathrm{X}]=0
$$

Where, $[\mathrm{k}]$ and $[\mathrm{m}]$ are stiffness and mass matrix for the human body, calculated from Equation 13, $\omega$ is the natural frequency for translational and rotational motion of the human body modelled as in Figure 3, and $[\mathrm{X}]$ is the normal modes for translational and rotational motion.

\section{BIOMECHANICAL FINITE ELEMENT MODEL}

The finite element analysis with the help of the COMSOL Multi-Physics Multi-body Dynamics interfaces package software has been used to model and simulate the dynamic biomechanics of human body behaviour. The dynamic response under various frequencies applied load, and the natural frequency for each part's human body is calculated. The biomechanical model using finite element technique is divided into different roles, 
first modelling for vibration behaviour for the human body, and secondly, meshes the human model with required element number by using mesh generation technique, and finally, solution vibration biomechanical human body model to evaluate the dynamic characterisations for modelling. The numerical results of the natural frequency, for each part of the human body, are found, comparison with analytical results calculating by using the analytical solution for biomechanical human body model, given in Equation 17, there is a good agreement between analytical and numerical results with a maximum error percentage $(4.2 \%)$.

\section{Human Body Vibration Model}

The biomechanical model of the human body has been modelled using the finite element method to estimate the vertical vibration parameters natural frequencies of the human body vibration. The model consists of all body parts; torso, head, pelvis, legs, thighs, and viscera, as illustrated in the model geometry for different body parts, connections, mass centre's, and vibration excitation locations (Figure 1). All the human body parts in this model are defined as rigid bodies and lumped masses. The moment of inertia and mass values for each part of the body are listed in Table 1 . The joints elasticity details for the human body parts in the model are shown in Table 2.

The fundamental governing equation for forced vibrations of a structure is written as Equation 16 (Rao, 2007; Rao, 2018),

$$
[\mathrm{m}]\{\ddot{\delta}\}+[\mathrm{c}]\{\dot{\delta}\}+[\mathrm{k}]\{\delta\}=\{\mathrm{F}\}
$$

Where, $[\mathrm{m}],[\mathrm{k}],[\mathrm{c}],\{\delta\}$, are the mass matrix, stiffness matrix, damping coefficient matrix, and displacement matrices, respectively, and is the external excitation force, as presented in Equation 14. Secondly, a frequency response analysis is modelled and carried out around the natural frequencies to determine the apparent mass and the two transmissibility. In sitting posture in vehicles, the vibration exciting the hip and thigh is transmitted to the human head through the entire body part. Therefore, the vibration transmissibility to the human head and driving point mechanical impedance or apparent mass of the human body are essential characteristics to express the vibration of a human body. The hip and human head vibration is also an important factor affecting ride comfort and visual disturbance. The vertical transmissibility (the ratio of vertical acceleration of the head to the input acceleration of the seat) is defined by Equation 17 (Kim et al., 2005),

$$
\mathrm{H}_{\text {vertical }}=\frac{(\ddot{y})_{\text {head }}}{(\ddot{y})_{\text {seat }}}
$$

The rotational transmissibility (the ratio of the angular acceleration of the head to the input acceleration of the seat) is defined by Equation 18 (Kim et al., 2005), 


$$
\mathrm{H}_{\text {rotational }}=\frac{(\ddot{\phi})_{\text {head }}}{(\ddot{y})_{\text {seat }}}
$$

The apparent mass (the ratio of the force at the seat to the input acceleration of the seat) is defined by Equation 19 (Kim et al., 2005),

$$
\mathrm{M}_{\text {apparent }}=\frac{(\mathrm{F})_{\text {seat }}}{(\ddot{\mathrm{y}})_{\text {seat }}}
$$

The transmissibility's and apparent mass are directly related to the comfort feeling. Especially the vertical and rotational transmissibility affect ride comfort and vision.

\section{Computational Grid}

The biomechanical model's governing equations were discretised using a finite-element method and solved using the COMSOL Multi-Physics Multi-body Dynamics interface package to simulate the different parts in the model. The human body segments and the whole-body vibrations are studied. Three different positions of a vertical direction excitation of $1 \mathrm{~m} / \mathrm{s}^{2}$ are achieved. Numerical testing was applied for these models, and a quadratic elements mesh domain, with 598 boundary elements was found to provide sufficient spatial resolution (Figure 5). The coupled set of equations of the human body model was solved iteratively. The solution was considered convergent when the relative error was less than $1.0 \times 10^{-6}$ in each field for two consecutive iterations.

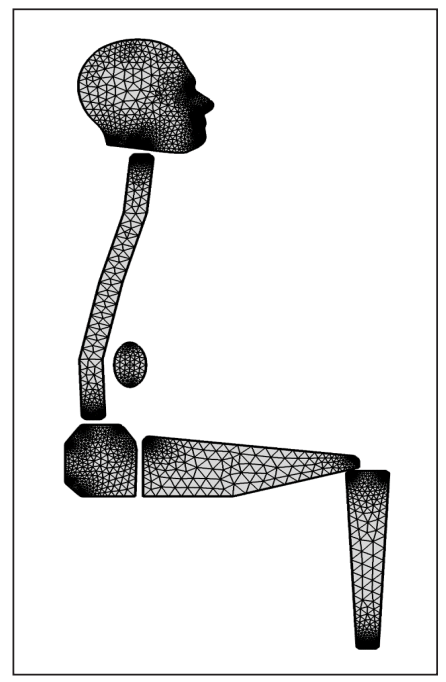

Figure 5. Computational quadratic mesh of the human body of the biomechanical model

\section{RESULTS AND DISCUSSION}

The biomechanical results included is employed to calculate the natural frequency for human body parts by using analytical and numerical techniques and then is compared the natural frequency results together, and after that, evaluating the vibration mode for human body parts with translational and rotational direction by using the numerical technique, by using finite element method.

\section{Natural Frequency Results}

The natural frequency for human body parts' translational and rotational biomechanical vibration is evaluated by an analytical solution for the general equation of motion for biomechanical modelling human body, Equation 15. Besides, the comparisons of the 
numerical results with analytical results were calculated, as shown in Table 6. The comparison of analytical and numerical results give a functional discrepancy with maximum error did not exceed about (4.2\%). Also, the results indicate that the maximum frequency occurs at a translational motion for the human body and the minimum frequency at rotational motion direction for the human body. So, these results can show the effect of applying load at the rotational human part more than the impact on the translational path. Furthermore, the results of the current work for both models were compared with the available published experimental results (Kim et al., 2005). The results showed that the maximum discrepancy did not exceed about (6\%), as in the Head rotation.

Table 6

Natural frequency for biomechanical human model general coordinate

\begin{tabular}{cccc}
\hline \multirow{2}{*}{ Human Model Coordinate } & \multicolumn{2}{c}{ Natural Frequency $(\mathrm{rad} / \mathrm{sec})$} & Discrepancy $(\%)$ \\
\cline { 2 - 3 } & Analytically & Numerically & 2.87 \\
$\mathrm{x}_{1}$ & 16.02 & 15.56 & 4.08 \\
$\mathrm{z}_{1}$ & 126.4 & 121.24 & 2.59 \\
$\theta_{1}$ & 76.56 & 74.58 & 4.56 \\
$\theta_{2}$ & 3.07 & 2.93 & 2.63 \\
$\theta_{3}$ & 29.24 & 28.47 & 3.05 \\
$\theta_{4}$ & 37.09 & 35.96 & 3.52 \\
$\theta_{5}$ & 25.01 & 24.13 & \\
\hline
\end{tabular}

\section{Numerical Results}

The graphical representation of the rotational Eigen-modes of the un-damped biomechanical model of the human body is explained in Figure 6. In this figure, the vast rotational movement of the head and torso segments related to the other parts of the human body (legs, pelvis, viscera, and thighs) was presented. Figure 7 shows the translational Eigen-mode of the damped biomechanical model of the human body. It also shows the vast movement of the viscera, pelvis, and head compared to the other parts of the human body (torso, legs, and thighs). The vertical transmissibility for a range of excitation frequencies is drawn in Figure 8, while Figure 9 shows the vertical transmissibility of the biomechanical model for a variety of excitation frequencies. Figure 10 shows the rotational transmissibility (deg/m) of the excitation frequency values of the biomechanical model. The rotational transmissibility reduces the comfort level, and also it directly affects the vision. Therefore, it is not good when it is increasing, especially at a higher value. Figure 11 shows the biomechanical model's apparent mass $(\mathrm{kg})$ for a range of excitation frequency. The results clearly show that the visible mass transfers the driving point physical appearance, concerning the motion and force at the seat. 
The present study results showed that the current two methodologies of the modelling and simulation of the human body using dynamic biomechanical vibration could sustain the design of vehicle seats. The mechanical response can be expected and predicted to respond to the human body using this biomechanical model in a vibratory environment as in a vehicle driving. It will help to stimulate the quality and design of vibration insulators such as seats, excitation frequency. The results clearly show that the visible mass transfers are associated with the driving point of physical appearance, the motion and the force at the seat.

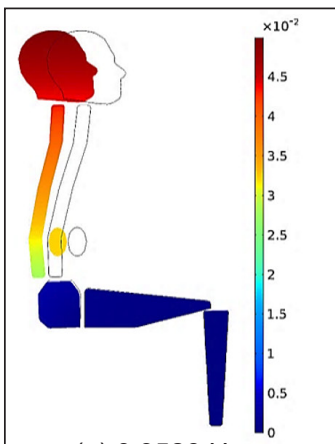

(a) $0.3526 \mathrm{~Hz}$

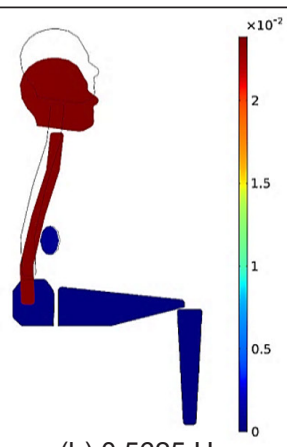

(b) $0.5625 \mathrm{~Hz}$

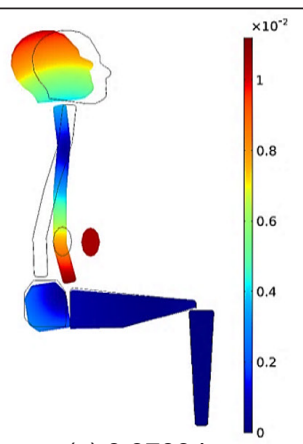

(c) $0.9709 \mathrm{hz}$

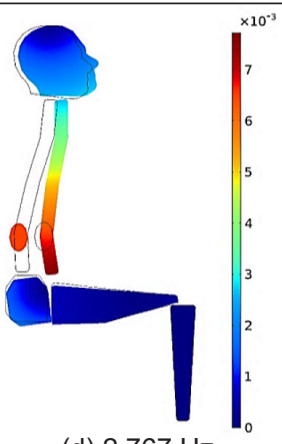

(d) $2.767 \mathrm{~Hz}$

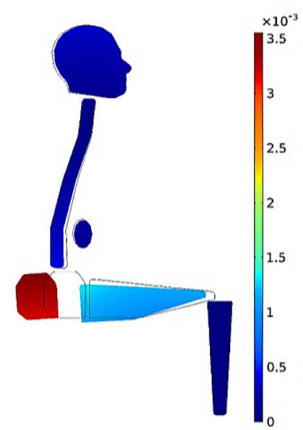

(e) $3.498 \mathrm{~Hz}$

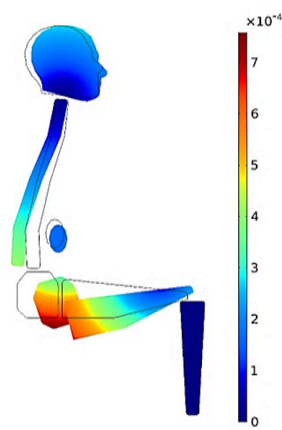

(f) $4.212 \mathrm{hz}$

(j) $8.231 \mathrm{~Hz}$

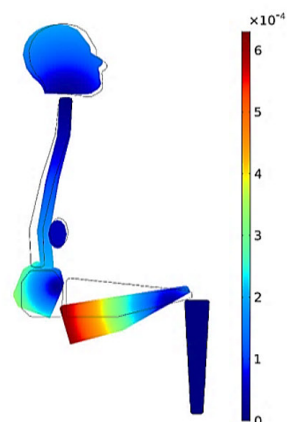

(g) $3.498 \mathrm{~Hz}$

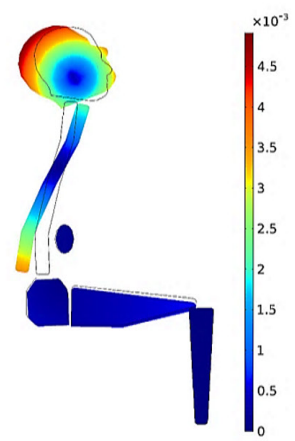

(k) $8.369 \mathrm{~Hz}$

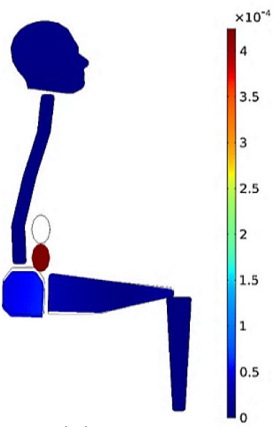

(h) $4.212 \mathrm{~Hz}$

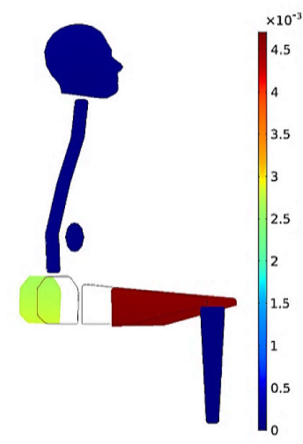

(i) $6.545 \mathrm{~Hz}$

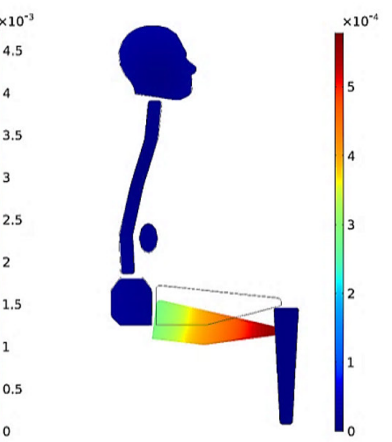

Figure 6. Rotational Eigen-Modes of the un-damped biomechanical model 


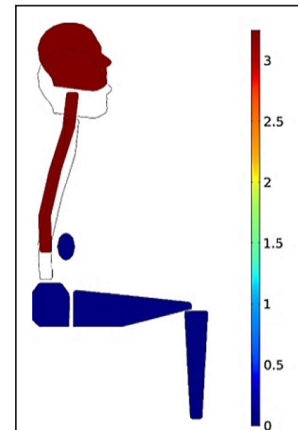

(a) $0.02665 \mathrm{i} \mathrm{Hz}$

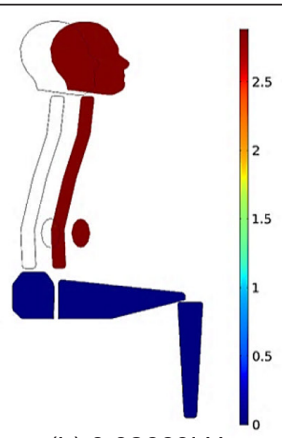

(b) $0.02669 \mathrm{i} \mathrm{Hz}$

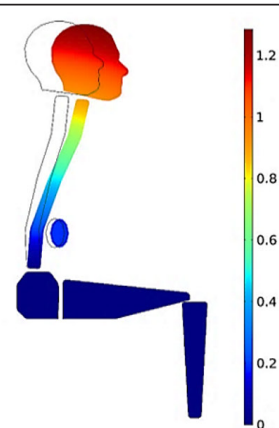

(c) $0.07242 \mathrm{i} \mathrm{Hz}$

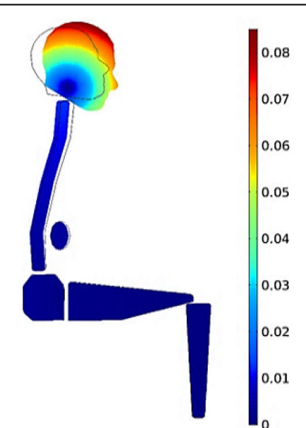

(d) $0.42980 \mathrm{i} \mathrm{Hz}$

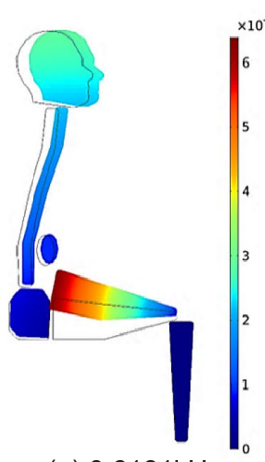

(e) $0.6181 \mathrm{i} \mathrm{Hz}$

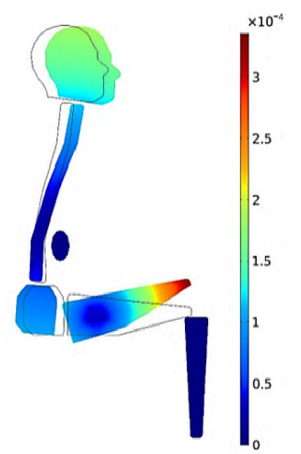

(f) $2.00200 \mathrm{ihz}$

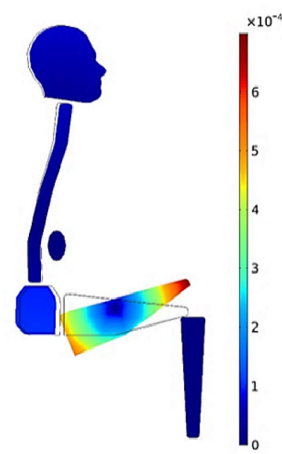

(g) $17.3500 \mathrm{i} \mathrm{Hz}$

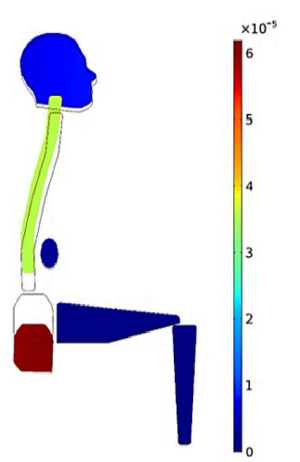

(h) $35.5600 \mathrm{i} \mathrm{Hz}$
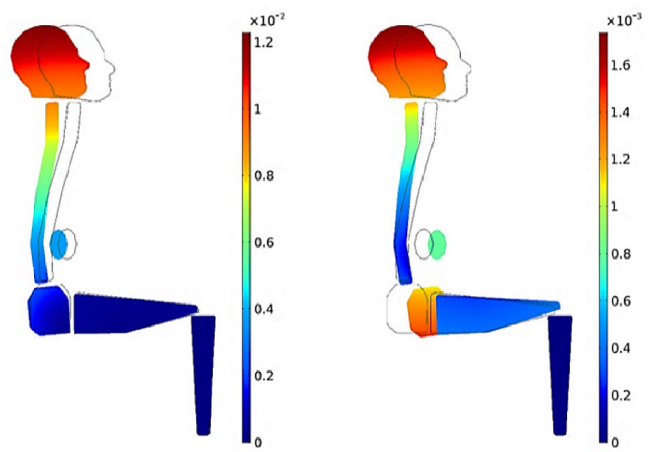

(j) $1.7180+0.4207 \mathrm{i} \mathrm{Hz}$

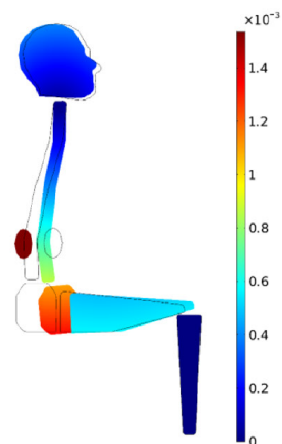

(k) $3.0630+0.7692 \mathrm{i} \mathrm{Hz}$

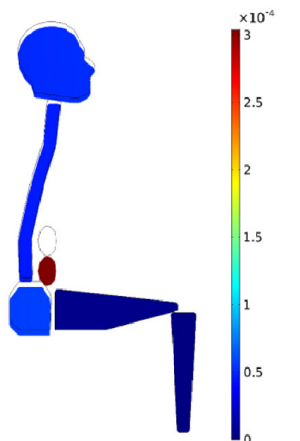

(I) $5.4090+0.8645 \mathrm{i} \mathrm{Hz}$
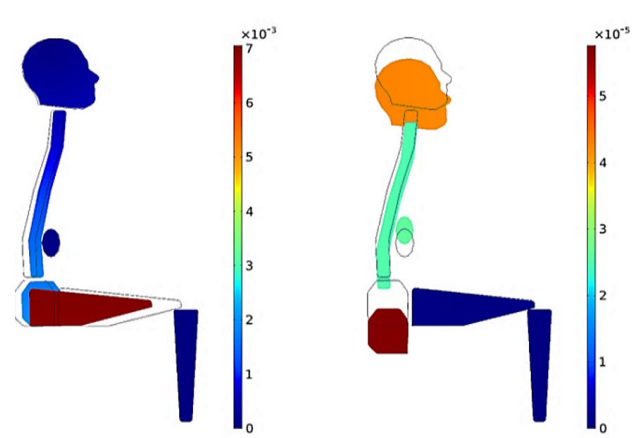

(n) $9.3320+4.4100 \mathrm{i} \mathrm{Hz}$

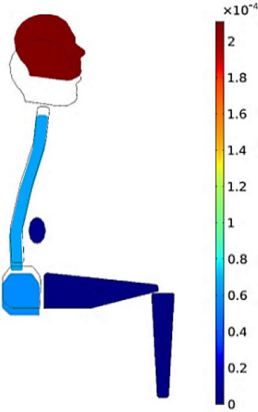

(o) $22.820+1.2290 \mathrm{i} \mathrm{Hz}$

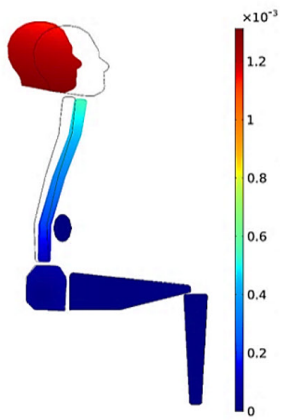

(p). $24.660+1.4180 \mathrm{i} \mathrm{Hz}$

Figure 7. Translational Eigen-Mode of the damped biomechanical model 


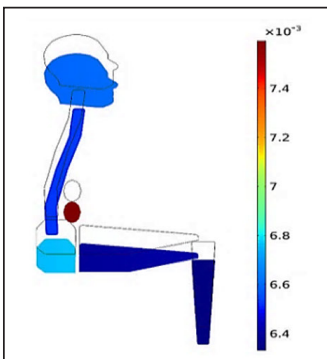

(a) $2 \mathrm{~Hz}$

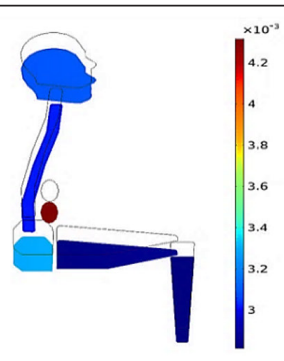

(b) $3 \mathrm{~Hz}$

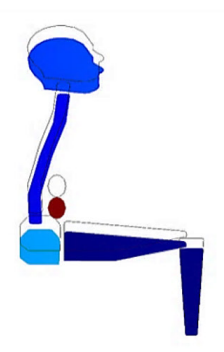

(c) $4 \mathrm{~Hz}$

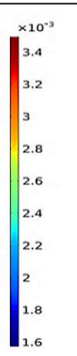

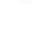

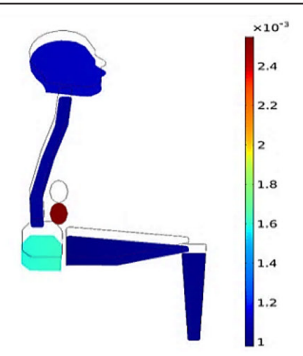

(d) $5 \mathrm{~Hz}$

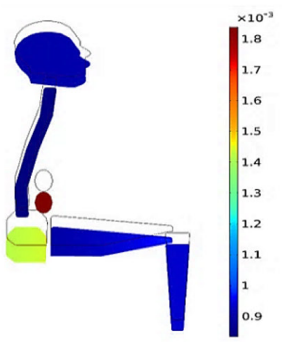

(e) $5.2 \mathrm{~Hz}$

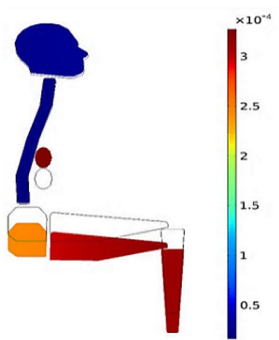

(i) $9 \mathrm{~Hz}$

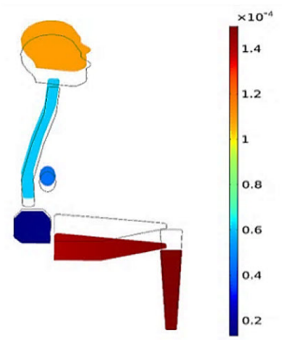

(i) $13 \mathrm{~Hz}$

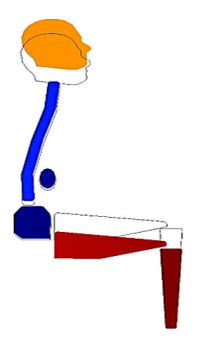

(m) $17 \mathrm{~Hz}$

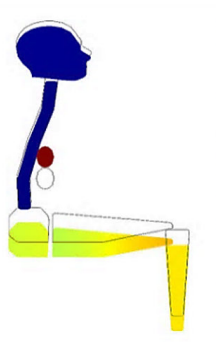

(f) $6 \mathrm{hz}$

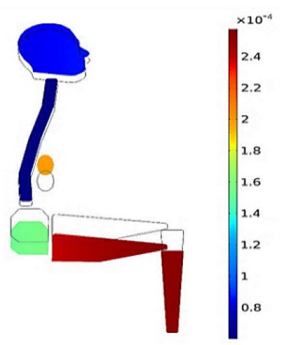

(j) $10 \mathrm{~Hz}$

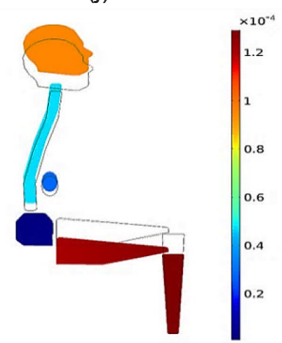

(j) $14 \mathrm{~Hz}$

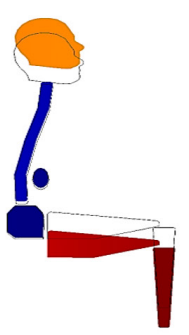

(n) $18 \mathrm{~Hz}$

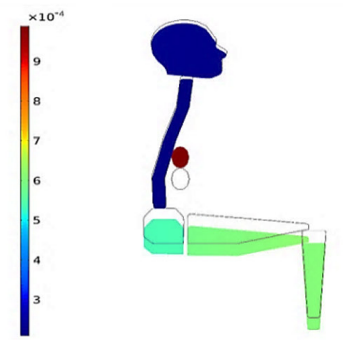

(g) $7 \mathrm{~Hz}$
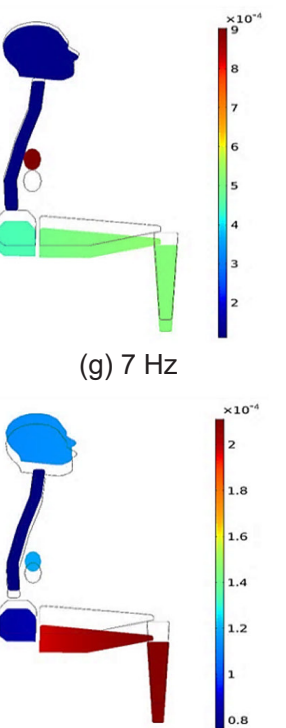

(k) $11 \mathrm{~Hz}$

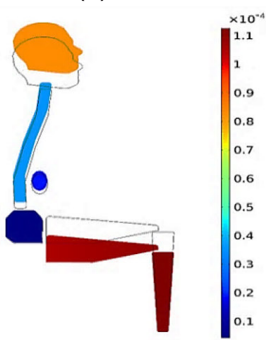

(k) $15 \mathrm{~Hz}$

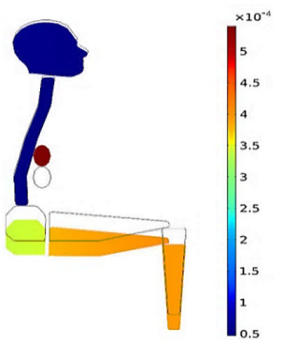

(h) $8 \mathrm{~Hz}$

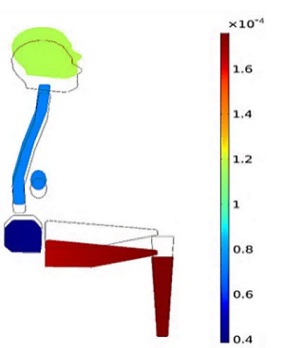

(I) $12 \mathrm{~Hz}$

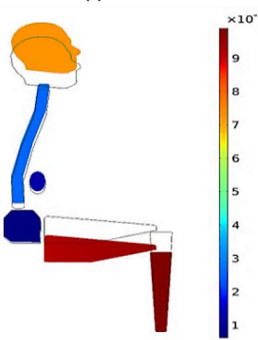

(I) $16 \mathrm{~Hz}$

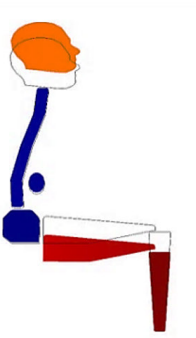

(o) $19 \mathrm{~Hz}$

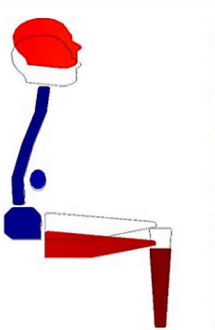

(p). $20 \mathrm{~Hz}$

Figure 8 . Vertical transmissibility for a range of excitation frequency 


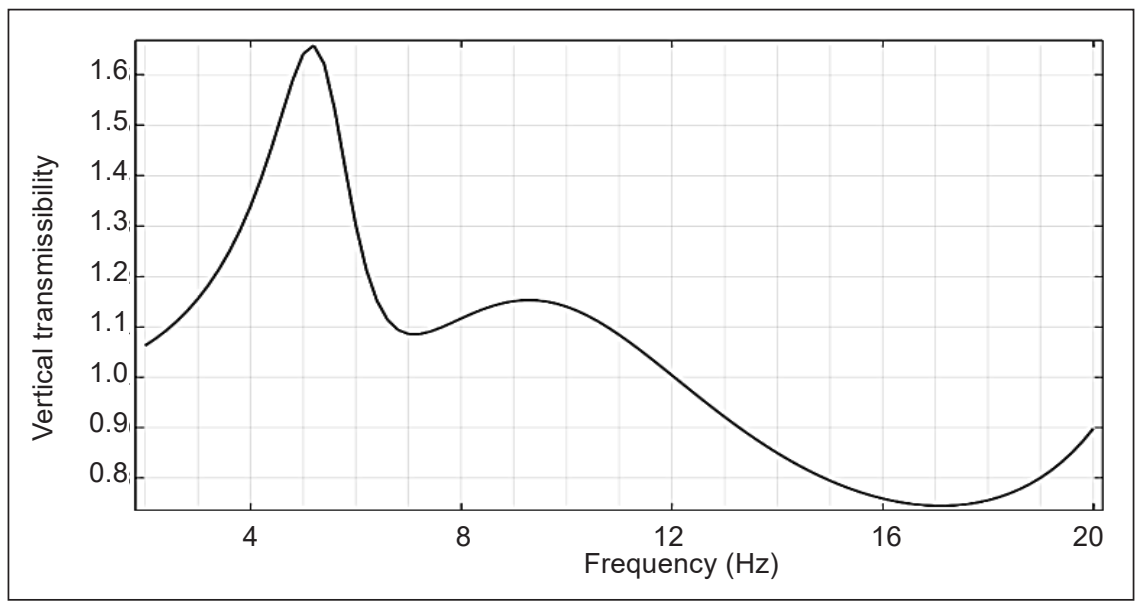

Figure 9. Vertical transmissibility of the biomechanical model for a range of excitation frequency

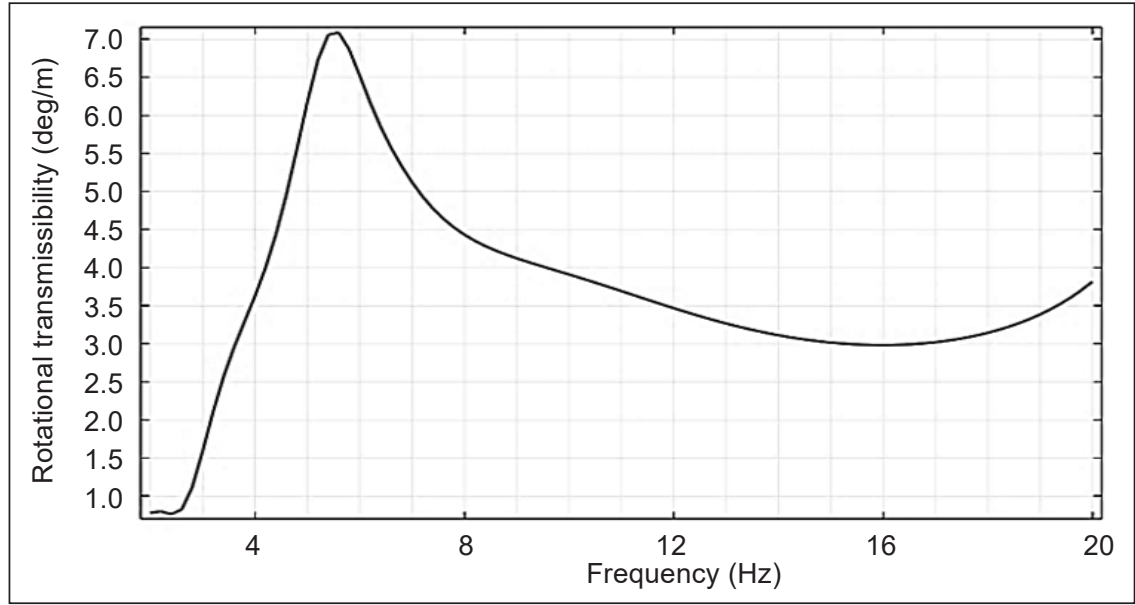

Figure 10. Rotational transmissibility of the biomechanical model for a range of excitation frequency

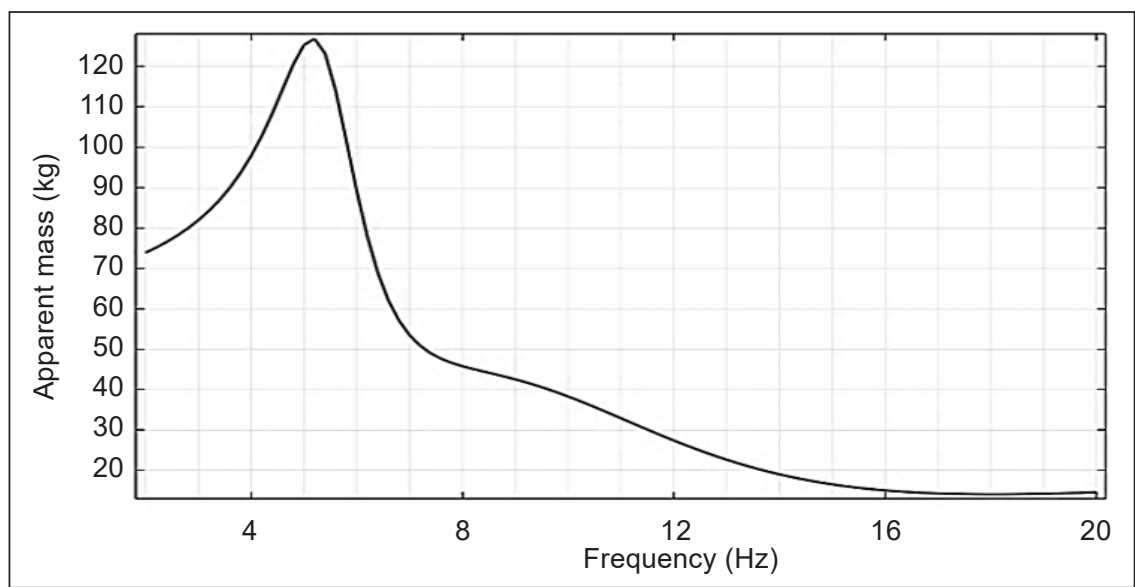

Figure 11. Apparent mass $(\mathrm{kg})$ of the biomechanical model for a range of excitation frequency 


\section{CONCLUSION}

In this study, analytical and numerical solutions for the dynamic biomechanical vibration model have been presented in detail to investigate and analyse the dynamic response of a human body when seated in a driving vehicle. The study included modelling the human body using a dynamic biomechanical vibration model and simulation with two methodologies. The first simulation was an analytical solution by deriving the general equations of the motion of the vibration behaviour for the human body to calculate the natural frequency for individual parts. The second simulation was a finite element technique to calculate natural frequency and vibration modes for the human body under different frequency loads. The simulation results show that the mechanical response expected and predicted to respond to the human body is perfect using this biomechanical model in a vibratory environment as in a vehicle driving, which will help stimulate the quality and design of vibration insulator such as a seat. Therefore, from this study can be concluded the following important points:

- The numerical vibration biomechanical human body model is a suitable technique and more sensitive that can be used to calculate the natural frequency for each part of the human body.

- Comparing human natural frequency results between analytical and numerical methods gives a good agreement results with maximum discrepancy not exceeding about $(4.2 \%)$, and not exceeding (6\%) compared with the available published experimental results.

- The maximum frequency occurs at a translational motion for the human body, and the minimum frequency at rotational motion direction for the human body occurs. Therefore, it can be shown that applying load on the rotational human parts is more than the effect of load on the translational human parts. So, the vibration response occurs at rotational human parts more than at translational human parts.

- The results are significant for human health in sitting position in the driving vehicle, which can assist understanding of the effects of low-frequency vibration on human health, comfort, and performance. Therefore it could be applied for ride comfort evaluation.

\section{ACKNOWLEDGEMENT}

The authors would like to thank the University of Kufa and the University of Al-Farahidi, Iraq, to support and fund this study.

\section{REFERENCES}

Abbas, H. J., Jweeg, M. J., Al-Waily, M., \& Diwan, A. A. (2019). Experimental testing and theoretical prediction of fiber optical cable for fault detection and identification. Journal of Engineering and Applied Sciences, 14(2), 430-438. https://doi.org/10.36478/jeasci.2019.430.438 
Al-Waily, M. (2005). Analysis of stiffened and unstiffened composite plates subjected to time dependent loading (MSc Thesis). University of Kufa, Iraq.

Bayoglu, R., Galibarov, P. E., Verdonschot, N., Koopman, B., \& Homminga, J. (2019). Twente spine model: A thorough investigation of the spinal loads in a complete and coherent musculoskeletal model of the human spine. Medical Engineering \& Physics, 68, 35-45. https://doi.org/10.1016/j.medengphy.2019.03.015

Desai, R., Guha, A., \& Seshu, P. (2018). Multibody biomechanical modelling of human body response to direct and cross axis vibration. Procedia Computer Science 133, 494-501. https://doi.org/10.1016/j. procs.2018.07.062

Griffin, M. J. (1990). Handbook of human vibration. Academic Press.

Guo, L. X., Dong, R. C., \& Zhang, M. (2016). Effect of lumbar support on seating comfort predicted by a whole human body-seat model. International Journal of Industrial Ergonomics, 53, 319-327. https://doi. org/10.1016/j.ergon.2016.03.004

ISO 2631-1. (1997). Mechanical vibration and shock - Evaluation of human exposure to whole-body vibration. International Organizational for Standardization

ISO 5982. (2001). Mechanical vibration and shock - Range of idealized values to characterize seated-body biodynamic response under vertical vibration. International Organizational for Standardization.

Kim, T. H., Kim, Y. T., \& Yoon, Y. S. (2005). Development of a biomechanical model of the human body in a sitting posture with vibration transmissibility in the vertical direction. International Journal of Industrial Ergonomics, 35, 817-829. https://doi.org/10.1016/j.ergon.2005.01.013

Koutras, C., Pérez, J., Kardash, K., \& Otaduy, M. A. (2021). A study of the sensitivity of biomechanical models of the spine for scoliosis brace design. Computer Methods and Programs in Biomedicine 207, Article 106125. https://doi.org/10.1016/j.cmpb.2021.106125

Mohanty, P. P., \& Mahapatra, S. S. (2014). A finite element approach for analyzing the effect of cushion type and thickness on pressure ulcer. International Journal of Industrial Ergonomics, 44, 499-509. https:// doi.org/10.1016/j.ergon.2014.03.003

Nimmen, K. V., Lombaert, G., Roeck, G. D., \& den Broeck, P. V. (2017). The impact of vertical human-structure interaction on the response of footbridges to pedestrian excitation. Journal of Sound and Vibration, 402, 104-121. https://doi.org/10.1016/j.jsv.2017.05.017

Rao, S. S. (2007). Vibration of continuous systems. John Wiley and Sons, Inc.

Rao, S. S. (2018). Mechanical vibrations. Pearson Education, Inc.

Vergari, C., Chen, Z., Robichon, L., Courtois, I., Ebermeyer, E., Vialle, R., Langlais, T., Pietton, R., \& Skalli, W. (2020). Towards a predictive simulation of brace action in adolescent idiopathic scoliosis. Computer Methods in Biomechanics and Biomedical Engineering, 24(8), 874-882. https://doi.org/10.1080/10255 842.2020 .1856373

Zheng, G., Qiu, Y., \& Griffin, M. J. (2011). An analytic model of the inline and cross-axis apparent mass of the seated human body exposed to vertical vibration with and without a backrest. Journal of Sound and Vibration, 330, 6509-6525. https://doi.org/10.1016/j.jsv.2011.06.026 